\title{
Numerical Study of Micropolar Fluids Flow due to a Stretching Cylinder by SOR Iterative Procedure
}

\author{
Atif Nazir ${ }^{1}$, Mohammad Shafique, ${ }^{2, *}$ \\ ${ }^{1}$ Mathematics Group Coordinator, Department of General Studies, Yanbu Industrial College, Saudi Arabia \\ ${ }^{2}$ Gomal University, D I Khan, Pakistan
}

Copyright $@ 2015$ Horizon Research Publishing All rights reserved.

\begin{abstract}
The steady flow of a micropolar fluid, due to a stretching cylinder, is considered. The equations of motion are reduced to a system of ordinary differential equations, which in turn are solved numerically using SOR iterative procedure and Simpson's (1/3) rule, for three different combinations of the three dimensionless parameters involved. Results are calculated, for the range 0.1 to 100 of the parameter $R$. The accuracy of the results was checked by comparing them on different grid sizes and with the previous results where possible.
\end{abstract}

Keywords Micropolar Fluids, Stretching Cylinder, Numerical Solution

AMS Subject Classification: 76D99, 76M20, 65N22

\section{Introduction}

The flow due to stretching boundaries has been investigated for various situations such as permeable/ impermeable medium, magnetohyderodynamic flows, heat transfer, stagnation point flow and non-Newtonian fluids flow. Eringen [1] introduced a class of fluids known as micro fluids. These fluids exhibit certain microscopic effects due to the local structure and micro-motions of the fluid elements. Later on, Eringen [2] offered the theory of micropolar fluids, a subclass of microfluids and established constitutive equations for fluids with micro-structure. This theory included the micro-rotational effects, micro-rotational inertia and couple stresses in addition to the usual stresses in the fluid medium. Physically this class of bar-like elements such as certain an isotropic fluids (e.g., liquid crystals, which are made up of dumbbell molecules), animal blood, polymeric fluids and fluids with certain additives. Using the basic equations derived in Eringen [2], Wilson [3] studied the two dimensional steady flow of the boundary layer type near a stagnation point with the help of Karman-Polhausen integral method. Peddieson and McNitt [4] investigated the steady stagnation point flow over a semi-infinite plate. Ebert [5] has obtained a similarity solution for boundary layer stagnation point flow.

The fluid flow due to a stretching surface is important in extrusion processes. Due to its practical importance, many investigators have studied this problem in detail for Newtonian fluids. Crane [6] first obtained the solution to two-dimensional stretching of a flat surface. Brady and Acrivos [7] investigated the similarity exact solution of the flow inside a stretching channel and inside a stretching cylinder. The three dimensional stretching of a rotating fluid was discussed by Wang [8]. The problem of the three dimensional flow due to a stretching flat surface has been solved by Wang [9]. He found as exact similarity solution of the Navier-Stokes equations which may be considered as the stretching counterpart of the exact stagnation point solution of Homann [10] and Howarth [11]. Wang [12] solved the problem of the exterior fluid flow due to the stretching cylinder. Sankara and Watson [13] investigated the steady micropolar flow past a stretching sheet. Crane [14] found the boundary layer solution outside a stretching cylinder. Watanabe and Oyama [15] studied maganatohydrodynamic boundary layer flow over a rotating disk. Dash and Tripathy [16] considered hydromagnatic fluid flow between two horizental plates, both the plates being stretching sheets. Datta et al. [17] studied the effect of non-uniform slot injection/suction on a forced flow over a slender cylinder. Ishak et al [18] studied the steady laminar magnetohydrodynamic (MHD) boundary-layer flow past a wedge with constant surface heat flux immersed in an incompressible micropolar fluid in the presence of a variable magnetic field. The steady mixed convection boundary layer flow over a vertical surface immersed in an incompressible micropolar fluid is considered in the paper of Ishak et al [19]. Two dimensional steady, laminar and incompressible motion of a micropolar fluid between an impermeable disk and a permeable disk is considered by Ashraf et al [20] to investigate the influence of the Reynolds number and the micropolar structure on the flow characteristics. Sajid et al [21] considered the boundary layer flow of a micropolar fluid through a porous channel. The flow occurs due to suction and injection at the walls of the channel. A finite 
element solution of the problem of heat and mass transfer in a hydromagnetic flow of a micropolar fluid past a stretching sheet is investigated by Kumar [22]. The flow of an incompressible micropolar fluid over a suddenly moved plate is considered under isothermal conditions by Devakar and Iyengar [23]. The boundary layer flow of a micropolar fluid due to a linearly stretching sheet is studied in the limit of a vanishing coupling parameter by Tarek [24]. An exact solution is obtained by Kumaran et al [25] for a boundary layer flow of an electrically conducting fluid past a quadratically stretching, and linearly permeable sheet. The steady, two-dimensional, stagnation point flow of an incompressible micropolar fluid over a stretching sheet when the sheet is stretched in its own plane with a velocity proportional to the distance from the stagnation point has been studied by Nazar et al [26].

Kamal and Hussain [27, 28] obtained the numerical results, using SOR method, for the micropolar fluid motion caused by the stretching of a surface in a rotating fluids and inside a stretching channel. Kamal, Ashraf and Syed [29] considered a two dimensional flow of a micropolar fluid driven by injection between two porous disks. Kamal and Siaft [30] investigated the stretching of a surface in a rotating micropolar fluid while Shafique and Rashid [31] obtained numerical solution of three dimensional micropolar flows due to a stretching flat surface.

The problem of Wang [12], for micropolar fluids is examined in the present work. To obtain the numerical solution of the problem under consideration, we used the similarity transformations to reduce the equations of motion to non-linear ordinary differential equations. The finite difference equations are obtained and solved by using SOR iterative procedure and the Simpson's (1/3) rule, subject to the appropriate boundary conditions. The numerical results are given in tabular as well as graphical form and are compared with the results of Newtonian fluids in a particular case.

There are many numerical techniques to solve the fluids flow problems but the finite difference and finite element schemes are the most prominent among them. Finite difference approximations are found to be discrete techniques wherein the domain of interest is represented by a set of points or nodes and information between these points is commonly obtained by using Taylor series expansions while the finite element method employs piecewise continuous polynomials to interpolate between nodal points. The finite difference techniques are very easy to understand and straight forward for computational analysis. However, it is not necessary to discuss the comparison of any other method like finite element with numerical solution of this problem.

\section{Mathematical Analysis}

The field equations of motion of micropolar fluids given by Eringen [2] are as under:

$$
\begin{gathered}
\frac{\partial \rho}{\partial t}+\operatorname{div}(\rho \underline{V})=0 \\
(\lambda+2 \mu+\kappa) \nabla(\nabla \cdot \underline{V})-(\mu+\kappa) \nabla \times \nabla \times \underline{V}+ \\
+\kappa \nabla \times \underline{v}-\nabla \pi+\rho f=\rho \underline{\dot{V}} \\
(\alpha+\beta+\gamma) \nabla(\nabla \underline{v})-\gamma(\nabla \times \nabla \times \underline{v})+ \\
+\kappa \nabla \times \underline{V}-2 \kappa \underline{v}+\rho l=\rho j \underline{\dot{v}} \\
\rho C_{p}\left(\frac{\partial T}{\partial \mathrm{t}}+(\underline{V} \cdot \nabla) T\right)=K \nabla^{2} T+\Phi
\end{gathered}
$$

where $\underline{V}$ is the fluid velocity vector, $\underline{v}$ is the micro-rotation vector, pthe density, $\pi$ the pressure, $f$ and $l$ are body force and body couple per unit mass respectively, $j$ the micro inertia, and $\lambda, \mu, \kappa, \alpha, \beta$ and $\gamma$ the material constants ( or viscosity coefficients). The dissipation function is $\Phi$ while $T, \mathrm{C}_{\mathrm{p}}$ and $K$ are fluid temperature, specific heat and heat conductivity respectively.

We consider the flow of micropolar fluid due to a stretching cylinder. In the analysis, we use the cylindrical coordinate system $(r, \theta, z)$ such that the cylinder is described with the radius $r=a$. We assume that the material constants of the micropolar fluid are independent of position and neglect body force and body couple. The $T_{w}$ represents the constant temperature of the surface of the tube and $T_{\infty}$ denotes the temperature of ambient fluid where $T_{w}>T_{\infty}$.

We, also, assume that the flow is steady, laminar and incompressible.

With these assumptions, the equations (1) to (4) become:

$$
\begin{gathered}
\nabla \cdot \underline{V}=0 \\
-(\mu+\kappa) \nabla \times \nabla \times \underline{V}+\kappa \nabla \times \underline{v}-\nabla \pi=\rho \underline{\dot{V}} \\
(\alpha+\beta+\gamma) \nabla(\nabla \cdot \underline{v})-\gamma(\nabla \times \nabla \times \underline{v})+ \\
+\kappa \nabla \times \underline{V}-2 \kappa \underline{v}=\rho j \underline{\underline{v}} \\
\rho C_{p}(\underline{V} \cdot \nabla) \mathrm{T}=K \nabla^{2} T .
\end{gathered}
$$

We look for a solution in which the velocity and microrotation vectors may be written in the form:

$$
\underline{V}=(u(r, z), 0, w(r, z)), \underline{v}=\left(0, v_{2}(r, z), 0\right) .
$$

The set of equations (5) to (8) in cylindrical coordinates system become:

$$
\begin{gathered}
\frac{\partial u}{\partial r}+\frac{u}{r}+\frac{\partial w}{\partial z}=0 \\
(\mu+\kappa)\left(\frac{\partial^{2} u}{\partial r^{2}}+\frac{1}{r} \frac{\partial u}{\partial r}+\frac{\partial^{2} u}{\partial z^{2}}-\frac{u}{r^{2}}\right)- \\
-\kappa\left(\frac{\partial \omega_{2}}{\partial z}\right)-\frac{\partial \pi}{\partial r}=\rho\left(u \frac{\partial u}{\partial r}+w \frac{\partial u}{\partial z}\right),
\end{gathered}
$$




$$
\begin{gathered}
(\mu+\kappa)\left(\frac{\partial^{2} w}{\partial r^{2}}+\frac{1}{r} \frac{\partial w}{\partial r}+\frac{\partial^{2} w}{\partial z^{2}}\right)+ \\
+\kappa\left(\frac{\omega_{2}}{r}+\frac{\partial \omega_{2}}{\partial r}\right)-\frac{\partial \pi}{\partial z}=\rho\left(u \frac{\partial w}{\partial r}+w \frac{\partial w}{\partial z}\right), \\
\kappa\left(\frac{\partial u}{\partial z}-\frac{\partial w}{\partial r}\right)-2 \kappa \omega_{2}-\gamma\left(\frac{\omega_{2}}{r^{2}}-\frac{\partial^{2} \omega_{2}}{\partial r^{2}}-\frac{1}{r} \frac{\partial \omega_{2}}{\partial r}-\frac{\partial^{2} \omega_{2}}{\partial z^{2}}\right) \\
=\rho j\left(u \frac{\partial \omega_{2}}{\partial r}+w \frac{\partial \omega_{2}}{\partial z}+\frac{u \omega_{2}}{r}\right), \\
\rho C_{p}\left(u \frac{\partial T}{\partial r}+w \frac{\partial T}{\partial z}\right)=K\left(\frac{1}{r} \frac{\partial T}{\partial r}+\frac{\partial^{2} T}{\partial r^{2}}+\frac{\partial^{2} T}{\partial z^{2}}\right) .
\end{gathered}
$$

Subject to the boundary conditions:

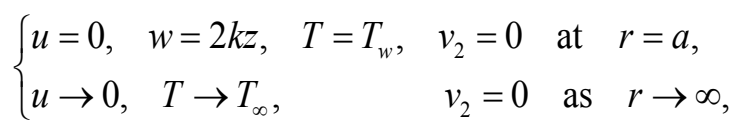

We now use the similarity transformations to make the equations of motion in dimensionless form as follows.

$$
\begin{aligned}
& u=-k a(f(\eta) / \sqrt{\eta}), w=2 k f^{\prime}(\eta) z, v_{2}= \\
& =-\frac{4 k}{a^{2}} r z L(\eta) \text { and } \theta=\frac{T-T_{\infty}}{T_{w}-T_{\infty}},
\end{aligned}
$$

where $\eta=(r / a)^{2}$ is the dimensionless variable.

If we substitute (15) in (9), then it may be verified that it is identically satisfied. The following equation can be determined from equation (10) as:

$$
\frac{\partial \pi}{\partial r}=\frac{\rho c^{2} a^{4}}{r^{3}} f^{2}-\frac{2 \rho c^{2} a^{2}}{r} f^{\prime}-\frac{4 \mu c r}{a^{2}} f^{\prime \prime} .
$$

The pressure $\pi$ can be obtained from this equation by integration with respect to $\eta$.

We use (15) into equations (11) to (13), then after simplification become:

$$
\begin{gathered}
\eta f^{\prime \prime \prime}+f^{\prime \prime}-C_{1}\left(L+\eta L^{\prime}\right)=R\left(f^{2}-f f^{\prime \prime}\right), \\
2 L^{\prime}+\eta L^{\prime \prime}+C_{2}\left(f^{\prime \prime}-2 L\right)= \\
=C_{3}\left(f^{\prime} L-f L^{\prime}-\eta^{-1} f L\right), \\
\eta \theta^{\prime \prime}+(1+R \operatorname{Pr} f) \theta^{\prime}=0,
\end{gathered}
$$

Where the prime denotes differentiation with respect to $\eta$. Here $R=\frac{k a^{2}}{2 v}$ is the Reynolds number, where $a$ is the radius of the cylinder, $v$ the coefficient of kinematics viscosity and $k$ the given constant of dimension [1/Time]. $\mathrm{C}_{1}$, $\mathrm{C}_{2}$ and $\mathrm{C}_{3}$ are all dimensionless constants given by

$$
\mathrm{C}_{1}=\frac{k}{\mu+k}, \mathrm{C}_{2}=\frac{k a^{2}}{4 \gamma}, \mathrm{C}_{3}=\frac{a^{2} \rho j \bar{k}}{2 \gamma} \text {. }
$$

The dimensions of the parameters involved are as follows:

$$
\begin{aligned}
& {[\mu, \kappa]=M T^{-1} L^{-1},[\gamma]=M L T^{-1},} \\
& {[j]=L^{2},[\rho]=M L^{-3},[k]=T^{-1} \text { and }[a]=L}
\end{aligned}
$$

In view of (15), the boundary conditions (14) take the form:

$$
\left\{\begin{array}{l}
f=0, \quad f^{\prime}=1, \quad \theta=1, \quad L=0, \quad \text { at } \quad \eta=1, \\
f^{\prime} \rightarrow 0, \quad \theta \rightarrow 0, \quad L \rightarrow 0, \quad \text { as } \quad \eta \rightarrow \infty .
\end{array}\right.
$$

It is interesting to note that the equations (16) and (17) reduce to the equation of motion for Newtonian fluids by vanishing micro rotation (or spin). The present results of Newtonian fluids are given in tables as well when spin is zero.

In order to solve numerically, it is convenient to reformulate the problem by using the following transformation:

$$
\eta=e^{x}
$$

Thus the equations (16) to (17) and boundary conditions (19), due to (20), become

$$
\begin{gathered}
f^{\prime \prime \prime}-2 f^{\prime \prime}+f^{\prime}-C_{1} e^{2 x}\left(L+L^{\prime}\right) \\
=R\left(f^{\prime 2}-f f^{\prime \prime}+f f^{\prime}\right) \\
L^{\prime}+L^{\prime \prime}+C_{2}\left(e^{-x}\left(f^{\prime \prime}-f^{\prime}\right)-2 e^{x} L\right) \\
=C_{3}\left(f^{\prime} L-f L^{\prime}-f L\right) \\
\theta_{x x}+R \operatorname{Pr} f(x) \theta_{x}=0,
\end{gathered}
$$

and

$$
\left\{\begin{array}{l}
f=0, \quad f_{x}=1, \quad \theta=1, \quad L=0, \quad \text { at } \quad x=0, \\
e^{-x} f_{x}=0, \quad \theta=0, \quad L=0, \quad \text { as } \quad x \rightarrow \infty,
\end{array}\right.
$$

where here the prime denotes differentiation with respect to $\mathrm{x}$.

In order to solve the equations (21) to (23) numerically, we set:

$$
P=f_{x}=f^{\prime}
$$

Then equations (21) and (22) become:

$$
\begin{aligned}
& \quad P^{\prime \prime}-2 P^{\prime}+P-C_{1} e^{2 x}\left(L+L^{\prime}\right) \\
& =R\left(P^{2}-f P^{\prime}+f P\right), \\
& L^{\prime}+L^{\prime \prime}+C_{2}\left(e^{-x}\left(p^{\prime}-p\right)-2 e^{x} L\right) \\
& =C_{3}\left(p L-f L^{\prime}-f L\right)
\end{aligned}
$$

The boundary conditions (24) become:

$$
\left\{\begin{array}{l}
f=0, \quad P=1, \quad \theta=1, \quad L=0, \quad \text { at } \quad x=0, \\
e^{-x} P \rightarrow 0, \quad \theta \rightarrow 0, \quad L \rightarrow 0, \quad \text { as } \quad x \rightarrow \infty .
\end{array}\right.
$$


Now, if we approximate the equation (23), (25) and (26) by central difference approximation at a typical point $\mathrm{x}=x_{\mathrm{n}}$ of the interval $[0, \infty)$, we obtain

$$
\begin{gathered}
\left(2-h^{2}+R h^{2} f_{n}+R h^{2} p_{n}\right) p_{n}-\left(\frac{R h f_{n}}{2}-h\right)\left(p_{n+1}-p_{n-1}\right)- \\
-\left(p_{n+1}+p_{n-1}\right)+\frac{h}{2} C_{1} e^{2 x}\left(L_{n+1}+L_{n-1}\right)+C_{1} h^{2} e^{2 x} L_{n}=0 \\
\left(C_{3} h^{2}\left(f_{n}-p_{n}\right)-2 C_{2} h^{2} e^{x}-2\right) L_{n}+\left(L_{n+1}+L_{n-1}\right)+ \\
+\left(\frac{h}{2} C_{3} f_{n}+\frac{h}{2}\right)\left(L_{n+1}-L_{n-1}\right)+\frac{h}{2} C_{2} e^{-x}\left(p_{n+1}-p_{n-1}\right)=0 \\
\left(2+h R \operatorname{Pr} f_{n}\right) \theta_{n+1}+ \\
+\left(2-h R \operatorname{Pr} f_{n}\right) \theta_{n-1}-4 \theta_{n}=0
\end{gathered}
$$

Where $h$ denotes a grid size, we shall replace the interval $[0, \infty)$ by $[0, t)$, where $t$ is a sufficiently large.

In order to solve numerically, the first order ordinary differential equations (25) and the finite difference equations (29) to (31) at each interior grid point of the interval, the equations (25) are integrated by the Simpson's (1/3) rule, with the formula given in Milne, whereas the equations (29) to (31) are solved by using SOR iterative procedure, subject to the boundary conditions (28). the computation has been checked for different of the relaxation parameter $\omega$ between 1 and 2 . The optimum value of the relaxation parameter for the problem under consideration is 1.5 . The SOR iterative procedure is terminated when the following criterion is satisfied:

$$
\max _{i=1}^{n}\left|U_{i}^{n+1}-U_{i}^{n}\right|<10^{-6}
$$

where $n$ denotes the number of iterations and $U$ stands for each of $P$ and $f$. and the accuracy of the solution has been checked for step sizes $h=0.01,0.005$ and 0.0025 and it is excellent. All numerical results are not given in next section due to reduce the number of pages of this paper. The comparison of numerical solutions is only given in next section for present results, Newtonian fluids and previous result of Wong [12].

\section{Discussion on Results}

The results obtained by using the numerical computation for the problem reported in the previous section are presented in tabular as well as graphical form. In order to analyze velocity, temperature, micro rotation, skin friction coefficient $f^{\prime \prime}(1)$ and the Nusselt number $-\theta^{\prime}(1)$, tables and curves have been drawn for representative values of the flow parameters namely $\mathrm{R}$ and $\mathrm{Pr}$. It is worth mentioning that accuracy of the results has been checked by computing the results on three different grid sizes namely $h=0.01,0.005$ and 0.0025 . Three different sets of the material constants $C_{1}, C_{2}$ and $\mathrm{C}_{3}$ given in the table below have been chosen arbitrarily and the results have been computed for each of the sets.

\begin{tabular}{|c|rcc|}
\hline Cases & $\mathrm{C}_{1}$ & $\mathrm{C}_{2}$ & $\mathrm{C}_{3}$ \\
\hline I & 0.1 & 0.2 & 0.3 \\
II & 0.05 & 0.1 & 0.2 \\
III & 0.02 & 0.15 & 0.25 \\
\hline
\end{tabular}

For reliability of the present results, an extensive comparison of these results with the results for Newtonian fluids and the previously published results is given in the Table 1 to Table 3 for $f^{\prime \prime}(1), f(\infty)$ and $-\theta^{\prime}(1)$ respectively. The results are in good agreement. It can be noticed from Table 1 that all the values of $f^{\prime \prime}(1)$ are negative. It means that a dragging force on the fluid is exerted by the stretching tube. The Prandtl number Pr has no effect on the velocity components $f$ and $f^{\prime}$.

Graphically the results have been reported for a representative set of all the parameters of interest in the form of velocity and temperature distributions. Figure 1 illustrates the effect of $R$ on $f$. The behavior of $f^{\prime}(\eta)$ under the effect of $R$ is shown in Figure 2. It is noticed that the velocity gradient at surface increases with increasing the values of $R$. Figure 3 and Figure 4 are drawn to observe the effect of $\mathrm{Pr}$ upon temperature function $\theta(\eta)$ for a fixed value of $R=10$. The curves have been presented in these figures for the values of $\operatorname{Pr}=0.7$ (air) and 7.0(water). In both the figures $\theta(\eta)$ decreases and then becomes zero at a large $\eta$ in both the cases. This situation causes the increase in wall temperature gradient and thus the surface heat transfer rate is increased.

Table 1. The comparison of Micropolar fluids and Newtonian fluids for $f^{\prime \prime}(1)$

\begin{tabular}{|c|c|c|c|c|c|}
\hline \multirow{2}{*}{$\mathrm{R}$} & \multicolumn{3}{|c|}{ Micropolar fluids } & \multicolumn{2}{c|}{ Newtonian fluids } \\
\cline { 2 - 6 } & $\mathrm{I}$ & $\mathrm{II}$ & III & Present (Spin is zero) & Wong [12] \\
\hline 0.1 & -0.5443344 & -0.4959164 & -0.4974709 & --- & ---- \\
\hline 0.5 & -0.8940945 & -0.8865318 & -0.8864365 & --- & ---- \\
\hline 1.0 & -1.1846790 & -1.1811456 & -1.1810026 & --- & ---- \\
\hline 2.0 & -1.5997895 & -1.5959939 & -1.5958842 & -1.595297 & -1.5941 \\
\hline 5.0 & -2.419663 & -2.410713 & -2.410623 & -2.410499 & -2.4175 \\
\hline 10.0 & -3.343893 & -3.318196 & -3.318130 & -3.318044 & -3.3445 \\
\hline 15.0 & -4.049064 & -4.007159 & -4.007102 & ---- & --- \\
\hline 20.0 & -4.645396 & -4.582964 & -4.583002 & ---- & --- \\
\hline
\end{tabular}


Table 2. The comparison of Micropolar fluids and Newtonian fluids for $f(\infty)$

\begin{tabular}{|c|c|c|c|c|c|}
\hline \multirow{2}{*}{$\mathrm{R}$} & \multicolumn{3}{|c|}{ Micropolar fluids } & \multicolumn{2}{c|}{ Newtonian fluids } \\
\cline { 2 - 6 } & $\mathrm{I}$ & $\mathrm{II}$ & $\mathrm{III}$ & Present (Spin is zero) & Wong [12] \\
\hline 0.1 & 5.03047 & 5.08722 & 5.17849 & ---- & ---- \\
\hline 0.5 & 2.58751 & 2.49823 & 2.51841 & --- & --- \\
\hline 1.0 & 1.72587 & 1.66509 & 1.67149 & --- & --- \\
\hline 2.0 & 1.13568 & 1.07165 & 1.07255 & 1.105823 & 1.0983 \\
\hline 5.0 & 0.59715 & 0.59275 & 0.59249 & 0.592566 & 0.5933 \\
\hline 10.0 & 0.38662 & 0.38595 & 0.38579 & 0.385362 & 0.3857 \\
\hline 15.0 & 0.30481 & 0.30354 & 0.30342 & --- & --- \\
\hline 20.0 & 0.25704 & 0.25719 & 0.25707 & -- & --- \\
\hline
\end{tabular}

Table 3. The comparison of Micropolar fluids and Newtonian fluids for $-\theta^{\prime}(1)$

\begin{tabular}{|c|c|c|c|c|c|}
\hline \multirow{2}{*}{$\operatorname{Pr}$} & \multicolumn{3}{|c|}{ Micropolar fluids } & \multicolumn{2}{c|}{ Newtonian fluids } \\
\cline { 2 - 6 } & I & II & III & Present (Spin is zero) & Wong [12] \\
\hline 0.1 & 1.035165 & 1.070427 & 1.058554 & --- & --- \\
\hline 0.7 & 1.696729 & 1.713347 & 1.713609 & 1.702547 & 1.5683 \\
\hline 2.0 & 3.028202 & 3.026080 & 3.027701 & 3.026962 & 3.0360 \\
\hline 7.0 & 6.155849 & 6.156290 & 6.155157 & 6.155753 & 6.1592 \\
\hline 10.0 & 7.462382 & 7.462192 & 7.461810 & 7.462454 & 7.4668 \\
\hline 15.0 & 9.258533 & 9.258176 & 9.257985 & --- & --- \\
\hline
\end{tabular}

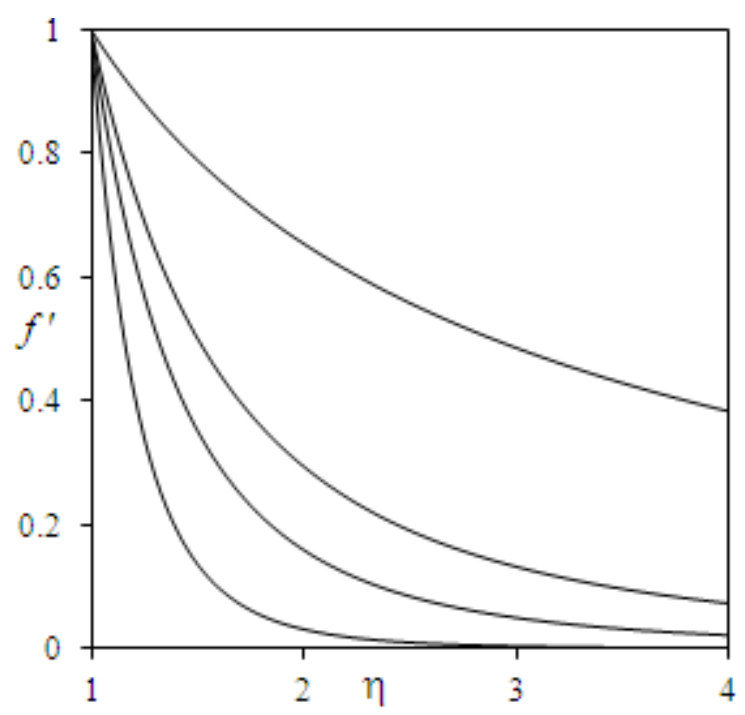

Figure 1. Graph of $f^{\prime}$ for the values of $R=0.1,2,5$ and 20 from top to bottom.

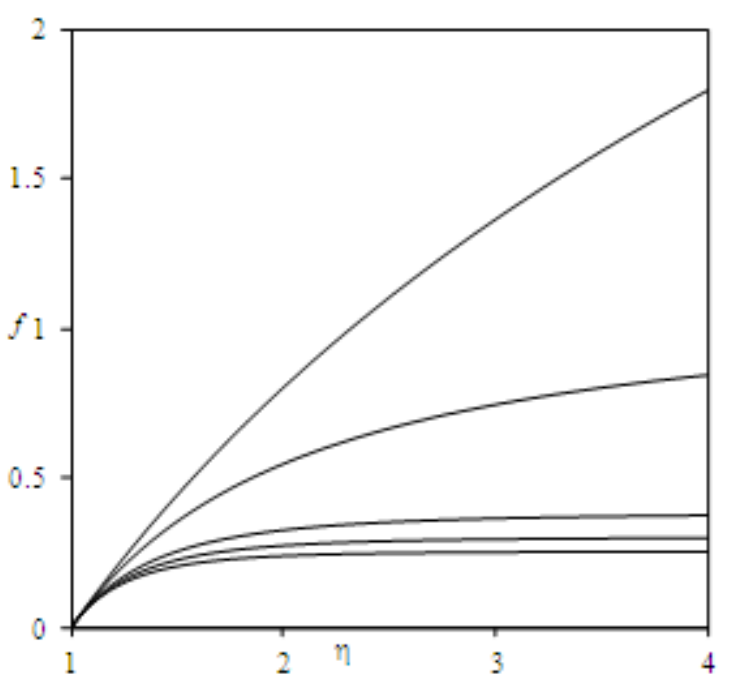

Figure 2. Graph of $f$ for the values of $R=0.1,2,5,10$ and 20 from top to bottom. 


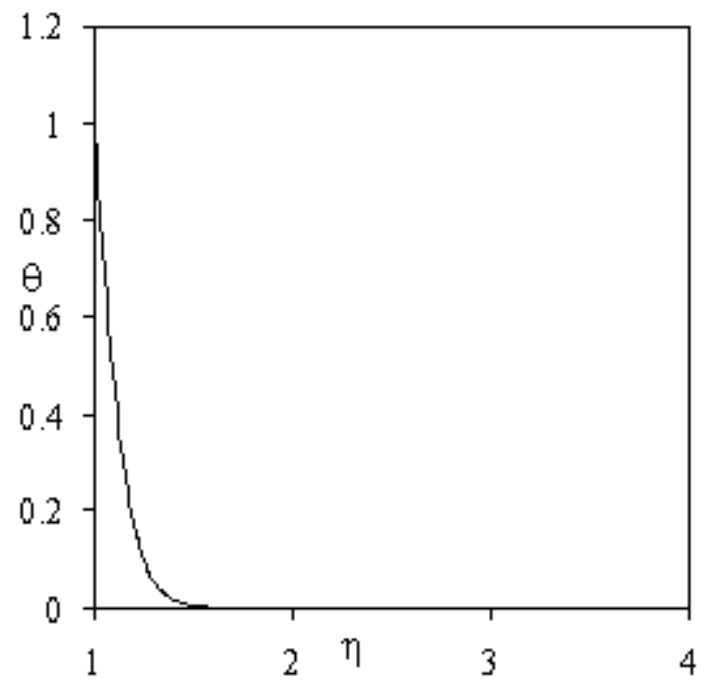

Figure 3. Graph of $\theta(\eta)$ for the values $\operatorname{Pr}=7, R=10$.

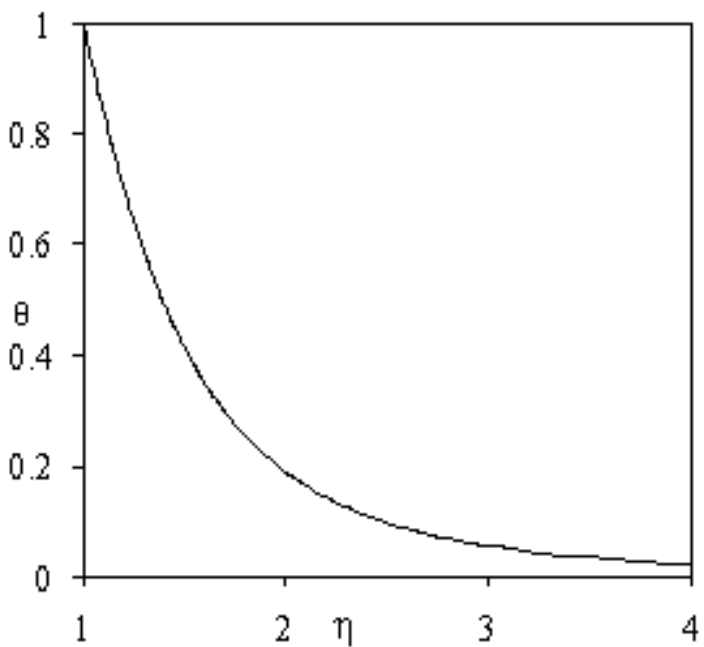

Figure 4. Graph of $\theta(\eta)$ for the values $\operatorname{Pr}=0.7, R=10$.

\section{REFERENCES}

[1] A.C. Eringen, Int. J. Engg. Sci., 2, 205-207, (1964).

[2] A.C. Eringen, J. Math. Mech., 16, 1-18, (1966).

[3] A.J. Wilson, Proc. Camb. Phil. Soc., 67, pp469, (1970).

[4] J. Peddieson and R.P. McNitt, Recent Adv. Engg. Sci., 5,405, (1970).

[5] F. Ebert, J. Chem. Engg., 5, pp85, (1973).

[6] L.J. Crane, Zeit. Angew. Math. phys., 21, 645-647, (1970).

[7] J.F. Brady and A. Acrivos, J. Fluid Mech., 112, 127-150, (1981).
[8] C.Y. Wang, Zeit. Angew. Math. phys., 39, 177-185,(1988).

[9] C.Y. Wang, Phys. Fluids, 27(8), 1915-1917, (1984).

[10] F. Homann, Zeit. Angew. Math. Mech., 16, pp153, (1936).

[11] I. Howarth, Phil. Mag., 42(7), pp1433, (1951).

[12] C.Y.Wang, J. Phys. Fluids, 31(3), pp466, (1988).

[13] Sankara, K. K. and Watson, L. T. (1985), ZAMP, 36.

[14] Crane, I.J. (1975), Zeit. Angew. Math. Phys., 26, pp619.

[15] Watanabe, T. and Oyama, T. (1992), ZAAM, 71(12), 522-524.

[16] Dash, G. C. and Tripathy, P. C. (1993), Modelling, Measurement and control, B, ASME Press, 51(2), 51-64.

[17] Datta, P., Anilkumar, D., Roy, S. and Mahanti, N.C. (2006), Int. J. Heat Mass Transfer, 49, 2366- 2371.

[18] A Ishak, R Nazar, I Pop, (2009) , Communications in Nonlinear Science and Numerical Simulation 14, 109-118.

[19] A Ishak, R Nazar, I Pop, (2009), Communications in Nonlinear Science and Numerical Simulation 14, 1324-1333.

[20] M Ashraf , M. A Kamal, K.S. Syed , (2009), Applied Mathematical Modelling 33, 1933-1943.

[21] Sajid M., Abbas Z.,Hayat T., (2009), Applied Mathematical Modelling 33, 4120-4125.

[22] Lokendra Kumar, (2009), Computational Materials Science $46,841-848$.

[23] Devakar M., Iyengar T.K.V., (2009), Applied Mathematical Modelling 33, 924-936.

[24] Tarek M.A. El-Mistikawy, (2009), European Journal of Mechanics B/Fluids 28, 253-258.

[25] Kumaran V., Banerjee A.K., Vanav Kumar A., Vajravelu K., (2009), Applied Mathematics and Computation 210, 26-32.

[26] R Nazar, N Amin, D Filip, I Pop, (2004), International Journal of Non-Linear Mechanics 39, 1227 - 1235.

[27] M.A. Kamal and S. Hussain, Int.J.Sci.Tech., spring-fall,30-36,(1994).

[28] M.A. Kamal and S. Hussain, J. Natural Sci. Math., 34(1), (1994).

[29] Kamal, M. A., Ashraf, M. and Syed, K. S. (2006), App Math. \& Comp,179, 1-10.

[30] Kamal, M.A. and Sifat, H. (1994), Int. J. of Sci. and Tech., Spring Hall, 30-36.

[31] Shafique, M. and Rashid, A. (2006), Int. J. of Math. analysis, 1(2),173-187.

[32] R.L. Burden, Numerical Analysis, Prindle, Weber \& Schmidt, Boston (1985). 